\title{
MX beamline environment for measuring accurate anomalous signals from light atoms
}

\author{
Naohiro Matsugaki ${ }^{\mathrm{a}}$, Masahide Hikita ${ }^{\mathrm{a}}$, Ayaka Harada ${ }^{\mathrm{c}}$, Yusuke Yamada ${ }^{\mathrm{a}}$, \\ Masahiko Hiraki ${ }^{b}$, Miki Senda ${ }^{c}$ and Toshiya Senda ${ }^{c}$ \\ aPhoton Factory, Structural Biology Research Center, 1-1 Oho, Tsukuba, Ibaraki \\ 3050801, Japan, naohiro.matsugaki@kek.jp \\ ${ }^{b}$ Mechanical Engineering Center, 1-1 Oho, Tsukuba, Ibaraki 3050801, Japan \\ 'Structural Biology Research Center, 1-1 Oho, Tsukuba, Ibaraki 3050801, Japan
}

Measuring weak anomalous signals from light atoms in macromolecular crystallography (MX) has experimental difficulties; longer wavelength X-ray is favorable to enhance anomalous diffraction, however, on the other hand more X-rays are absorbed in the beam path from the sample crystal to the detector which deteriorates the quality of the diffraction data. MX data collection is normally performed with the X-ray wavelength below $2 \AA$

Photon Factory $\mathrm{MX}$ beamline $\mathrm{BL}-1 \mathrm{~A}$ is routinely operated for the experiment with long wavelength X-ray from $2.7 \AA$ to $3.3 \AA$ [1]. It allows diffraction experiment completely under helium atmosphere, so that the absorption occurred in the beam path is negligible. Combined with the crystal processing technique using the deep UV laser facility, systematic errors due to $X$-ray absorption by the sample itself can be minimized [2]. Users are required to prepare samples with the standard manner which are then processed into appropriate sizes and shapes to obtain the maximum anomalous signals at the target wavelength. Applications to native SAD phasing will be presented as well as indications of the wavelength to be used.

\section{References}

[1] Liebschner D. et al. (2016). Acta Cryst., D72, 728-741.

[2] Basu S. et al. (2019). IUCrJ, 6. 